\title{
Stellar populations in radio galaxies
}

\author{
D. Raimann ${ }^{1,2,3}$, T. Storchi-Bergmann ${ }^{1}$, H. Quintana ${ }^{4}$, \\ R. Hunstead ${ }^{5}$ and L. Wizotski ${ }^{6}$ \\ ${ }^{1}$ Instituto de Física, UFGRS, Porto Alegre, RS, Brazil email:raimann@if.ufrgs.br \\ ${ }^{2}$ UERGS, Porto Alegre, RS, Brazil \\ ${ }^{3}$ UNOCHAPECÓ, Chapecó, SC, Brazil \\ ${ }^{4}$ Facultad de Física, Pontificia Universitad Catolica de Chile, Santiago, Chile \\ ${ }^{5}$ School of Physics, University of Sydney, Sydney, Australia \\ ${ }^{6}$ Universität Potsdam, Potsdam, Germany
}

\begin{abstract}
We characterize the stellar population of a complete sample of the closest southern radio-galaxies - 12 Fanaroff-Riley I (FR I), 8 FR II and 4 with undefined FRx type - and a comparison sample of 18 non-active elliptical and SO galaxies. We measured equivalent widths and continuum colours and their radial variations, and performed spectral synthesis as a function of distance from the nucleus. Only 4 of the radio-galaxies have more than $10 \%$ of the total flux at $4020 \AA$ contributed from 100 Myr old or younger stellar population components - including a featureless continuum due to an AGN. All 4 are FRII or FRx. The main difference between the stellar population of the radio-galaxies and that of the non-active ones is that the former have a larger contribution of the intermediate age component, suggesting a relation between the present radio activity and a past episode of star formation which occurred about 1 Gyr ago.
\end{abstract}

\section{Introduction}

The relation between recent star-formation and nuclear activity in galaxies has been the subject of a number of recent studies. Cid Fernandes \& Terlevich (1995) were the first to argue that the blue continuum in Seyfert 2 galaxies was mostly due to the contribution of young stars. Many other works have subsequently shown that young stars are frequently found in the nuclear region of Seyfert galaxies (Raimann et al. 2001, 2003 and references therein). In more luminous objects, Aretxaga et al. (2001) and Wills et al. (2004) found young and/or intermediate stellar populations in the nuclear regions of some nearby radio-galaxies, as well as Tadhunter et al. (2002) and Wills et al. (2002) for intermediate redshift ones.

A number of questions are, nevertheless, still open regarding the hosts of AGNs, and one of fundamental nature is if there are any systematic differences between the circumnuclear stellar population of the hosts of AGNs and those of non-active galaxies of the same Hubble type. In this work, we answer this question for a sample formed by the closest 24 radio-galaxies and a comparison sample of 18 non-active elliptical and SO galaxies.

\section{Observations and Spectral Synthesis}

Long slit spectra covering the spectral range $\approx 3600-7000 \AA$ have been obtained using the ESO NTT telescope for the radio galaxies and the $1.52 \mathrm{~m}$ telescope for the closer non-active galaxies with a slit of $1.5^{\prime \prime}$ and spectral resolution of $\approx 5 \AA$.

The spectral synthesis was performed using the probabilistic formalism described in Cid Fernandes et al. (2001). We reproduce the observed equivalent widths and continuum 

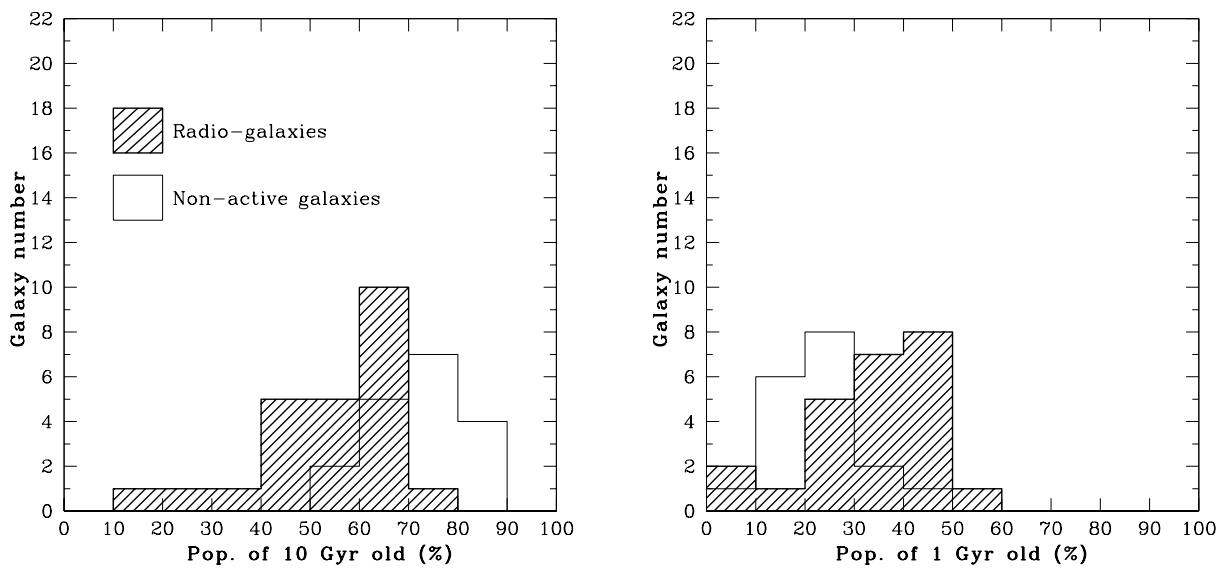

Figure 1. Radio-galaxies vs. non-active galaxies of the same Hubble type: galaxy number distribution according to the percentage contribution of the 10 and 1 Gyr populations to the total flux at $\lambda 4020 \AA$.

ratios using a base of star cluster spectra with different ages and metallicities (Bica \& Alloin 1986). We use 12 components representing the age- metallicity plane plus a $13^{\text {th }}$ component representing a cannonical AGN continuum $\mathrm{F}_{\nu} \propto \nu^{-1.5}$ (Schmitt et al. 1999).

\section{Results and Conclusions}

Only 4 radio galaxies have significant contribution ( $>10 \%$ of the total flux at $4020 \AA$ ) of stars younger than $1 \mathrm{Gyr}$ and/or of a featureless continuum; none of them is an FRI radio galaxy; 2 (of 8) FRII radio galaxies have broad lines in emission, thus at least part of the blue continuum is due to the AGN; one FRx radio galaxy has a significant contribution from the $100 \mathrm{Myr}$ old population; the stellar population of the radio-galaxies does not change significantly within the inner $5 \mathrm{kpc}$ or so.

In summary, we conclude that the fraction of low redshift radio galaxies $(z<0.08)$ with recent star formation and/or significant light from the AGN is about 10\% (3 out of 24 objects). In FRII/? radio-galaxies, this fraction increases to $23 \%$, what points to a small difference in the stellar population of FRI and FRII/? radio-galaxies.

Comparing the stellar population of the radio-galaxies with that of the non-active galaxies, we conclude that the contribution of the 10Gyr old population is on average $20 \%$ larger in non-active galaxies than in radio galaxies while that of the $1 \mathrm{Gyr}$ old population is $15 \%$ smaller (Fig. 1).

\section{References}

Aretxaga, I., et al. 2001, MNRAS, 325, 636

Bica, E., \& Alloin, D. 1986, A\&A, 162, 21

Canalizo, G., \& Stockton, A. 2001, ApJ, 555, 719

Cid Fernandes, R., \& Terlevich, R. 1995, MNRAS, 272, 423

Cid Fernandes, R., et al. 2001, ApJ, 558, 81

Raimann, D., et al. 2001, MNRAS, 324, 1087

Raimann, D., et al. 2003, MNRAS, 339, 772

Schmitt, H., et al. 1999, MNRAS, 303, 173

Tadhunter, C., et al. 2002, MNRAS, 330, 977

Wills, K. A., et al. 2002, MNRAS, 333, 211

Wills , K. A., et al. 2004, MNRAS, 347, 771 